Pacific Journal of Mathematics

ON THE LOCATION OF THE ZEROS OF CERTAIN
COMPOSITE POLYNOMIALS 


\title{
ON THE LOCATION OF THE ZEROS OF CERTAIN COMPOSITE POLYNOMIALS
}

\author{
AbDul AzIz
}

Let $P(z)=\sum_{j=0}^{n} C(n, j) A_{j} z^{j}$ and $Q(z)=\sum_{j=0}^{m} C(m, j) B_{j} z^{j}$ be two polynomials of degree $n$ and $m$, respectively, $m \leq n(C(n, j)=$ binomial coefficient). In this paper we study the relative location of the zeros of $P(z)$ and $Q(z)$ when the coefficients of these polynomials satisfy an apolar type relation and obtain some results. As an application of these results, we present certain generalizations of results of Walsh, Szegö, DeBruijn and Kakeya.

More recently [1] the author has shown that if

$$
P(z)=\sum_{j=0}^{n} C(n, j) A_{j} z^{j}, \quad A_{0} A_{n} \neq 0,
$$

and

$$
Q(z)=\sum_{j=0}^{m} C(m, j) B_{j} z^{j}, \quad B_{0} B_{m} \neq 0,
$$

are two polynomials of degree $n$ and $m$, respectively, $m \leq n$, such that

$$
\begin{aligned}
C(m, 0) B_{0} A_{n}-C(m, 1) B_{1} A_{n-1} \\
+\cdots+(-1)^{m} C(m, m) B_{m} A_{n-m}=0
\end{aligned}
$$

then the following holds.

(a) If $Q(z)$ has all its zeros in $|z| \geq r$, then $P(z)$ has at least one zero in $|z| \geq r$.

(b) If $P(z)$ has all its zeros in $|z| \leq r$, then $Q(z)$ has at least one zero in $|z| \leq r$.

Here we first show that this result equally holds if the circle $|z|=r$ is replaced by a more general circle $C$ with center at a point $c$ and radius $r$. In fact, we prove

THEOREM 1. If $P(z)$ is a polynomial of degree $n$ defined by (1) and $Q(z)$ is a polynomial of degree $m$ defined by (2), $m \leq n$, end if there coefficients satisfy the relation (3), then the following holds.

(i) If $Q(z)$ has all its zeros in $|z-c| \geq r$, then $P(z)$ has at least one zero in $|z-c| \geq r$. 
(ii) If $P(z)$ has all its zeros in $|z-c| \leq r$, then $Q(z)$ has at least one zero in $|z-c| \leq r$.

For the proof of Theorem 1, we need the following lemma, which is a generalization of a result due to Markovitch [4, p. 64].

LemMa. Let $P(z)=\sum_{j=0}^{n} C(n, j) A_{j} z^{j}$ and $Q(z)=\sum_{j=0}^{m} C(m, j) B_{j} z^{j}$ be two polynomials of degree $n$ and $m$, respectively, $m \leq n$. If we form

$$
U(z)=\sum_{j=0}^{n}(-1)^{j} P^{(n-j)}(z) Q^{(j)}(z)
$$

then

$$
U(z)=n ! \sum_{j=0}^{n}(-1)^{j} C(m, j) A_{n-j} B_{j} .
$$

Proof of the Lemma. Since $P(z)$ and $Q(z)$ are two polynomials of degree $n$ and $m$, respectively, we have

$$
P^{(n+1)}(z)=0 \text { and } Q^{(m+1)}(z)=0 .
$$

Now we can write

$$
P(z)=\sum_{j=0}^{n} C(n, j) A_{j} z^{j}=\sum_{j=0}^{n} \frac{P^{(j)}(0)}{j !} z^{j}
$$

and

$$
Q(z)=\sum_{j=0}^{n} C(m, j) B_{j} z^{j}=\sum_{j=0}^{n} \frac{Q^{(j)}(0)}{j !} z^{j},
$$

from which it follows that

$$
P^{(j)}(0)=C(n, j) j ! A_{j}, \quad j=0,1,2, \ldots, n,
$$

and

$$
Q^{(j)}(0)=C(m, j) j ! B_{j}, \quad j=0,1,2, \ldots, m
$$

Now

$$
\begin{aligned}
U^{\prime}(z)= & \sum_{j=0}^{n}(-1)^{j} P^{(n-j+1)}(z) Q^{(j)}(z) \\
& +\sum_{j=0}^{n}(-1)^{j} P^{(n-j)}(z) Q^{(j+1)}(z),
\end{aligned}
$$


so that by (4) we have

$$
\begin{aligned}
U^{\prime}(z)= & \sum_{j=1}^{m}(-1)^{j} P^{(n-j+1)}(z) Q^{(j)}(z) \\
& +\sum_{j=0}^{m-1}(-1)^{j} P^{(n-j)}(z) Q^{(j+1)}(z) \\
= & \sum_{j=0}^{m-1}(-1)^{j+1} P^{(n-j)}(z) Q^{(j+1)}(z) \\
& +\sum_{j=0}^{m-1}(-1)^{j} P^{(n-j)}(z) Q^{(j+1)}(z) \\
= & 0 .
\end{aligned}
$$

Therefore, it follows that $U(z)$ is constant and thus

$$
U(z)=U(0)=\sum_{j=0}^{m}(-1)^{j} P^{(n-j)}(0) Q^{(j)}(0) .
$$

Using (5) and (6) we obtain

$$
U(z)=n ! \sum_{j=0}^{m}(-1)^{j} C(m, j) A_{n-j} B_{j} .
$$

This proves the lemma.

Proof of Theorem 1. Consider

$$
H(z)=P(z+c)=\sum_{j=0}^{n} \frac{P^{(j)}(c)}{j !} z^{j}=\sum_{j=0}^{n} C(n, j) A_{j}^{*} z^{j} \quad \text { (say) }
$$

and

$$
G(z)=Q(z+c)=\sum_{j=0}^{m} \frac{Q^{(j)}(c)}{j !} z^{j}=\sum_{j=0}^{m} C(m, j) B_{j}^{*} z^{j} \quad \text { (say). }
$$

Then $H(z)$ and $G(z)$ are two polynomials of degree $n$ and $m$, respectively, $m \leq n$. Now we have

$$
\begin{aligned}
\sum_{j=0}^{m}( & -1)^{j} C(m, j) A_{n-j}^{*} B_{j}^{*} \\
& =\sum_{j=0}^{m}(-1)^{j} C(m, j) \frac{P^{(n-j)}(c)}{C(n, n-j)(n-j) !} \frac{Q^{(j)}(c)}{C(m, j) j !} \\
& =\frac{1}{n !} \sum_{j=0}^{m}(-1)^{j} P^{(n-j)}(c) Q^{(j)}(c) .
\end{aligned}
$$


Using the lemma above, we obtain

$$
\begin{aligned}
\sum_{j=0}^{m}(-1)^{j} C(m, j) A_{n-j}^{*} B_{j}^{*} & =\sum_{j=0}^{m}(-1)^{j} C(m, j) A_{n-j} B_{j} \\
& =0 \text { (by hypothesis). }
\end{aligned}
$$

This shows that the coefficients of the polynomials $H(z)$ and $G(z)$ satisfy (3). Hence it follows from (a) and (b) that if all the zeros of $G(z)$ lie in $|z| \geq r$, then $H(z)$ has at least one zero in $|z| \geq r$ and if all the zeros of $H(z)$ lie in $\mid z \leq r$, then $G(z)$ has at least one zero in $|z| \leq r$. Replacing $z$ by $z-c$ and noting that $P(z)=H(z-c)$ and, $Q(z)=G(z-c)$, the conclusion of Theorem 1 follows immediately.

As the first application of Theorem 1, we shall prove the following result, which is a generalization of Walsh's Coincidence Theorem [5] for the case when the circular region $C$ is a circle $|z-c|=r$.

THEOREM 2. Let $G\left(z_{1}, z_{2}, \ldots, z_{n}\right)$ be a symmetric n-linear form of total degree $m, m \leq n$, in $z_{1}, z_{2}, \ldots, z_{n}$ and let $C:|z-c| \leq r$ be a circle containing the $n$ points $w_{1}, w_{2}, \ldots, w_{n}$. Then in $C$ there exists a least one point w such that

$$
G(w, w, \ldots, w)=G\left(w_{1}, w_{2}, \ldots, w_{n}\right)
$$

Proof of Theorem 2. We write

$$
P(z)=\prod_{j=1}^{n}\left(z-z_{j}\right)=\sum_{j=0}^{n} C(n, j) A_{j} z^{j} \quad(\text { say }),
$$

so that

$$
C(n, j) A_{n-j}=(-1)^{j} S(n, j) A_{n}, \quad j=1,2, \ldots, n,
$$

where $S(n, j)$ are the symmetric functions consisting of the sum of all possible products of $z_{1}, z_{2}, \ldots, z_{n}$ taken $j$ at a time.

Now if $G\left(w_{1}, w_{2}, \ldots, w_{n}\right)=G^{*}$, then the difference $G\left(z_{1}, z_{2}, \ldots, z_{n}\right)$ $-G^{*}$ is linear, symmetric and of total degree $m \leq n$, in the variables $z_{1}, z_{2}, \ldots, z_{n}$. So that by the well-known theorem of algebra, $G\left(z_{1}, z_{2}, \ldots, z_{n}\right)-G^{*}$ can be expressed as a linear combination of the elementary symmetric functions $S(n, j), j=0,1, \ldots, m$. That is, there 
exist constants $B_{j}$ so that

$$
\begin{aligned}
& G\left(z_{1}, z_{2}, \ldots, z_{n}\right)-G^{*} \\
& \quad=B_{0}+S(n, 1) B_{1}+S(n, 2) B_{2}+\cdots+S(n, m) B_{m} \\
& \quad=\frac{1}{A_{n}}\left\{B_{0} A_{n}-C(n, 1) B_{1} A_{n-1}+\cdots+(-1)^{m} C(n, m) B_{m} A_{n-m}\right\}
\end{aligned}
$$

by using (7).

If we define the polynomial $Q(z)$ by

$$
Q(z)=\sum_{j=0}^{m} C(m, j) \frac{C(n, j)}{C(m, j)} B_{j} z^{j}=G(z, z, \ldots, z)-G^{*},
$$

then the relation

$$
G\left(w_{1}, w_{2}, \ldots, w_{n}\right)-G^{*}=0
$$

shows that the polynomials $P(z)$ and $Q(z)$ satisfy the condition of Theorem 1. Since all the zeros of $P(z)$ lie in $|z-c| \leq r$, we conclude from the 2nd part of Theorem 1 that $Q(z)=G(z, z, \ldots, z)-G^{*}$ has at least one zero in the circle $|z-c| \leq r$. This is equivalent to the desired result.

We next apply Theorem 1 to deduce the following partial generalization of a result due to Szegö [4, p. 65].

THEOREM 3. If all the zeros of a polynomial $Q(z)=\sum_{j=0}^{m} C(m, j) B_{j} z^{j}$ of degree $m$ lie in $|z-c| \geq r$ and if $\alpha$ is a zero of the polynomial $P(z)=$ $\sum_{j=0}^{n} C(n, j) A_{j} z^{j}, A_{0} A_{n} \neq 0$, of degree $n, m \leq n$, then every zero $w$ of the polynomial $R(z)=\sum_{j=0}^{m} C(m, j) A_{j} B_{j} z^{j}$ of degree $m$, has the form $w=$ $-\alpha \beta$, where $\beta$ is a suitably chosen point in $|z-c| \geq r$.

Proof of Theorem 3. If $w$ is a zero of $R(z)$, then

$$
R(w)=\sum_{j=0}^{m} C(m, j) A_{j} B_{j} w^{j}=0 .
$$

Equation (8) shows that the polynomials

$$
\begin{aligned}
z^{n} P(-w / z)= & C(n, 0)(-1)^{n} A_{n} w^{n}+\cdots+C(n, m)(-1)^{m} A_{m} w^{m} z^{n-m} \\
& +\cdots+C(n, n) A_{0} z^{n}
\end{aligned}
$$

and

$$
Q(z)=C(m, 0) B_{0}+C(m, 1) B_{1} z+\cdots+C(m, m) B_{m} z^{m}
$$

satisfy the conditions of Theorem 1 . Since all the zeros of $Q(z)$ lie in $|z-c| \geq r$, it follows from the first part of Theorem 1 that $z^{n} P(-w / z)$ 
has at least one zero in $|z-c| \geq r$. If $\alpha_{1}, \alpha_{2}, \ldots, \alpha_{n}$ are the zeros of $P(z)$, then the zeros of $z^{n} P(-w / z)$ are $-w / \alpha_{1},-w / \alpha_{2}, \ldots,-w / \alpha_{n}$. One of these zeros must be $\beta$, where $|\beta-c| \geq r$. Hence we must have $w=-\alpha_{j} \beta$ for some $j=1,2, \ldots, n$. This completes the proof of Theorem 3 .

REMARK 1. We may use the 2 nd part of Theorem 1 to establish Theorem 3 of [1] exactly in the same way as above for the case when the disk $|z| \leq r$ is replaced by more general disk $|z-c| \leq r$.

We shall now prove the following theorem, which is a generalization of a result due to DeBruijn [2].

THEOREM 4. From the two given polynomials

$$
P(z)=\sum_{j=0}^{n} C(n, j) A_{j} z^{j} \text { and } Q(z)=\sum_{j=0}^{m} C(m, j) B_{j} z^{J}
$$

of degree $n$ and $m$, respectively, $m \leq n$, let us form the third polynomial

$$
R(z)=\sum_{j=0}^{m} C(m, j) A_{j} B_{j} z^{j}
$$

of degree $m$. Given a subset $S$ of the $w$ plane, let $P(z) \in S$ for $|z| \leq r$ and $Q(z) \neq 0$ for $|z|<1$. Then $R(z) \in B_{0} S$ for $|z| \leq r$ where

$$
B_{0} S=\left\{B_{0} s ; s \in S\right\} \text {. }
$$

Proof of Theorem 4. Let $\delta$ be a real or a complex number. We replace the polynomial $P(z)$ by the polynomial $F(z)=P(z)-\delta$ and hence $R(z)$ by $H(z)=R(z)-\delta B_{0}$. If $\delta \notin S$, then $F(z)$ does not vanish in $|z| \leq r$. So that all the zeros of $F(z)$ lie in $|z|>r$. Also, by hypothesis, the zeros of $Q(z)$ lie in $|z| \geq 1$. Now if $w$ is a zero of $H(z)$, then by Theorem 2 of [1], $w$ has the form $w=-\alpha \beta$ where $\alpha$ is a suitably chosen point in $|z|>r$ and $\beta$ is a zero of $Q(z)$. Hence $|w|=|\alpha||\beta| \geq|\alpha|>r$. This shows $H(z)$ does not vanish in $|z| \leq r$. If therefore, $\delta B_{0}$ is a value assumed by $R(z)$ in $|z| \leq r$, then $\delta$ is a value assumed by $P(z)$ in $|z| \leq r$. Since $P(z) \in S$ for $|z| \leq r$, it follows that $R(z) \in B_{0} S$ for $|z| \leq r$ and this completes the proof.

Remark 2. Let $P(z), Q(z)$ and $R(z)$ be as in Theorem 4, and $S$ be a subset of the $w$ plane. Let $Q(z) \in S$ for $|z| \leq r$ and $P(z) \neq 0$ for $|z|<1$. Then by using Theorem 3 with $c=0$, it can be shown, similarly as above, that $R(z) \in A_{0} S$ for $|z| \leq r$, where $A_{0} S=\left\{A_{0} s ; s \in S\right\}$.

As an application of Theorem 4 and Remark 2, we deduce the following result which is also a generalization of another result of DeBruijn [2]. 
COROLlaRY 1. If the polynomials $P(z)$ and $Q(z)$ defined in Theorem 4 have the properties

$$
|P(z)| \leq 1 \text { and }|Q(z)| \leq 1 \text { for }|z| \leq 1,
$$

then the polynomial $R(z)$ of Theorem 4 has the property

$$
|R(z)| \leq 1-|| A_{0}|-| B_{0}|| \text { for }|z| \leq 1 .
$$

Proof of Corollary 1. Let $\alpha$ be a complex number such that $|\alpha|>1$. Then the polynomial $G(z)=(Q(z)-\alpha) /\left(B_{0}-\alpha\right)$ does not vanish in $|z| \leq 1$. Since $|P(z)| \leq 1$ for $|z| \leq 1$, we use Theorem 4 with $S:|z| \leq 1$, $r=1, Q(z)$ replaced by $G(z)$, and $R(z)$ by $\left(R(z)-\alpha A_{0}\right) /\left(B_{0}-\alpha\right)$. Hence it follows that

$$
\left|R(z)-\alpha A_{0}\right| \leq\left|B_{0}-\alpha\right| \text { for }|z| \leq 1 .
$$

This gives

$$
|R(z)| \leq\left|\alpha A_{0}\right|+\left|B_{0}-\alpha\right| \text { for }|z| \leq 1
$$

and for all $\alpha$ with $|\alpha|>1$.

Since $\left|B_{0}\right|=|Q(0)| \leq 1$, we can choose an argument of $\alpha$ such that $\left|B_{0}-\alpha\right|=|\alpha|-\left|B_{0}\right|$. Using this in (9) and letting $|\alpha| \rightarrow 1$ we get

$$
|R(z)| \leq 1+\left|A_{0}\right|-\left|B_{0}\right| \text { for }|z| \leq 1 .
$$

If we take $F(z)=(P(z)-\alpha) /\left(A_{0}-\alpha\right),|\alpha|>1$ and use the above remark, we can similarly show that

$$
|R(z)| \leq 1+\left|B_{0}\right|-\left|A_{0}\right| \text { for }|z| \leq 1 .
$$

The required result now follows by combining (10) and (11) and the proof is complete.

As another application of Theorem 1, we shall next prove the following generalization of a result due to Walsh [5].

THEOREM 5. From the two given polynomials

$$
P(z)=\sum_{j=0}^{n} a_{j} z^{j}=a_{n} \prod_{j=1}^{n}\left(z-\alpha_{j}\right)
$$

and

$$
Q(z)=\sum_{j=0}^{m} b_{j} z^{j}=b_{m} \prod_{j=1}^{m}\left(z-\beta_{j}\right)
$$

of degree $n$ and $m$, respectively, $m \leq n$, let us form the thir polynomial

$$
R(z)=\sum_{j=0}^{m}(n-j) ! a_{n-j} Q^{(j)}(z)
$$


of degree $m$. Then the following holds:

(i) If all the zeros of $P(z)$ lie in a circle $C:|z-c| \leq r$, then all the zeros of $R(z)$ lie in the point set $S$ consisting of the $m$ circular regions obtained by translating $C$ in the magnitude and direction of the vectors $\beta_{j}$.

(ii) If all the zeros of $Q(z)$ lie in the circular region $A:|z-c| \geq r$, then every zero $w$ of $R(z)$ has the form $w=\beta+\alpha$ where $\beta$ is a suitably chosen point in $A$ and $\alpha$ is a zero of $P(z)$.

Proof of Theorem 5. If $w$ is any zero of $R(z)$, then

$$
R(w)=\sum_{j=0}^{m}(n-j) ! a_{n-j} Q^{(j)}(w)=0 .
$$

Equation (12) shows that the polynomials

$$
P(z)=\sum_{j=0}^{n} a_{j} z^{j} \text { and } Q(w-z)=\sum_{j=0}^{m}(-1)^{j} \frac{Q^{(j)}(w)}{j !} z^{j}
$$

of degree $n$ and $m$, respectively, $m \leq n$, satisfy the conditions of Theorem 1. Since all the zeros of the polynomial $P(z)$ lie in $|z-c| \leq r$, it follows from the 2nd part of Theorem 1 that the polynomial $Q(w-z)$ has at least one zero in $|z-c| \leq r$. But the zeros of $Q(w-z)$ are $w-\beta_{1}$, $w-\beta_{2}, \ldots, w-\beta_{m}$. One of these zeros must be $\alpha$, where $|\alpha-c| \leq r$. That is, we must have $w=\alpha+\beta_{j}$ for some $j=1,2, \ldots, m$, where $\alpha$ is a point of $C$. This completes the proof of the first part of the Theorem.

To prove the 2 nd part of the theorem, we observe that the polynomial $R(z)$ can also be written in the form

$$
R(z)=\sum_{j=0}^{m}(n-j) ! a_{n-j} Q^{(j)}(z)=\sum_{j=0}^{m}(m-j) ! b_{m-j} P^{(n-m+j)}(z),
$$

so that if $w$ is a zero of $R(z)$, then we have

$$
R(w)=\sum_{j=0}^{m}(m-j) ! b_{m-j} P^{(n-m+\jmath)}(w)=0 .
$$

This equation shows that the polynomials

$$
P(w-z)=\sum_{j=0}^{n}(-1)^{J} \frac{P^{(j)}(w)}{j !} z^{j} \text { and } Q(z)=\sum_{j=0}^{m} b_{j} z^{j}
$$

also satisfy the conditions of Theorem 1 . Since all the zeros of $Q(z)$ lie in $|z-c| \geq r$, it follows from the first part of Theorem 1 that $P(w-z)$ has at least one zero in $|z-c| \geq r$. But the zeros of $P(w-z)$ are $w-\alpha_{1}$, $w-\alpha_{2}, \ldots, w-\alpha_{n}$, therefore, it folflows that for at least one $j=$ $1,2, \ldots, n$, we have $w=\alpha_{j}+\beta$, where $\beta$ is a suitably chosen point in $A$. This is equivalent to the desired result. 
Exactly in the same way as Theorem 5, we may deduce the following result from Theorem 1 of [1].

THEOREM 6. From the two given polynomials

$$
P(z)=\sum_{j=0}^{n} a_{j} z^{j}=a_{n} \prod_{j=1}^{n}\left(z-\alpha_{j}\right)
$$

and

$$
Q(z)=\sum_{j=0}^{m} b_{j} z^{j}=b_{m} \prod_{j=1}^{m}\left(z-\beta_{j}\right)
$$

of degree $n$ and $m$, respectively, $m \leq n$, let us form the polynomials

$$
R_{1}(z)=\sum_{j=0}^{m} \frac{(m-j) !(n-m+j) !}{j !} a_{m-j} Q^{(j)}(z)
$$

of degree $m$ and

$$
R_{2}(z)=\sum_{j=0}^{m}(n-j) ! b_{m-j} P^{(j)}(z)
$$

of degree $n$. Then we have the following:

(i) If all the zeros of $P(z)$ lie in $|z| \geq r$, then every zero $w$ of $R_{1}(z)$ has the form $w=\alpha+\beta$, where $\alpha$ is a sutiably chosen point in $|z| \geq r$ and $\beta$ is a zero of $Q(z)$.

(ii) If all the zeros of $Q(z)$ lie in $|z| \leq r$, then every zero $w$ of $R_{2}(z)$ has the form $w=\alpha+\beta$, where $\beta$ is a suitably chosen point in $|z| \leq r$ and $\alpha$ is a zero of $P(z)$.

As an immediate consequence of Theorems 5 and 6 we have the following corollary, which presents a generalization of a result of Kakeya [3].

COROLlaRY 2. If all the zeros of a polynomial $P(z)=\sum_{j=0}^{n} a_{j} z^{j}$ of degree $n$ lie in $|z| \leq r_{1}$ and all the zeros of a polynomial $Q(z)=\sum_{j=0}^{m} b_{j} z^{j}$ of degree $m$ lie in $|z| \leq r_{2}$, then all the zeros of the polynomials

$$
H(z)=\sum_{j=0}^{m}(n-j) ! a_{n-j} Q^{(j)}(z)
$$

of degree $m$ and

$$
R(z)=\sum_{j=0}^{m}(n-j) ! b_{m-j} P^{(j)}(z)
$$

of degree $n$ lie in $|z| \leq r_{1}+r_{2}$. 
This follows from the fact that $|\alpha| \leq r_{1}$ and $|\beta| \leq r_{2}$ imply $|w|=$ $|\alpha+\beta| \leq r_{1}+r_{2}$.

\section{REFERENCES}

[1] A. Aziz, On the zeros of composite polynomials, Pacific J. Math., 103 (1982), 1-7.

[2] N. G. DeBruijn, Inequalities concerning polynomials in the complex domain, Nederl. Akad. Wetensch. Proc., 50 (1947), 1265-1272.

[3] S. Kakeya, On algebraic equations having the roots of limited magnitude, Proc. Phys.-Math. Soc. Japan (3), 3 (1921), 94-100.

[4] M. Mardan, Geometry of Polynomials, 2nd ed., Mathematical Surveys, no. 3, Amer. Math. Soc., Providence, R. I. 1966.

[5] J. L. Walsh, On the location of the roots of certain types of polynomials, Trans. Amer. Math.. Soc., 24 (1922), 163-180.

Received August 17, 1983.

UNIVERSITY OF KASHMIR

HAZRATBal SRinagar - 190006

KASHMIR, INDIA 


\title{
PACIFIC JOURNAL OF MATHEMATICS EDITORS
}

DONALD BABBITT (Managing Editor)
University of California
Los Angeles, CA 90024
CHARLEs R. DEPrIMA
California Institute of Technology
Pasadena, CA 91125
R. FINN
Stanford University
Stanford, CA 94305

DoNAld BABBitT (Managing Editor)

Hermann FlaschKa

University of Arizona

Tucson, AZ 85721

RAmesh A. Gangolli

University of Washington

Seattle, WA 98195

ROBION KIRBY

University of California

Berkeley, CA 94720

C. C. MOORE

University of California

Berkeley, CA 94720

\author{
Hugo Rossi \\ University of Utah \\ Salt Lake City, UT 84112 \\ H. SAMELSON \\ Stanford University \\ Stanford, CA 94305 \\ HAROLD STARK \\ University of California, San Diego \\ La Jolla, CA 92093
}

ASSOCIATE EDITORS

R. ARENS

E. F. BECKENBACH
(1906-1982)

B. H. NEUMANN

F. WOLF

K. Yoshida

\section{SUPPORTING INSTITUTIONS}

UNIVERSITY OF ARIZONA

UNIVERSITY OF BRITISH COLUMBIA

CALIFORNIA INSTITUTE OF TECHNOLOGY

UNIVERSITY OF CALIFORNIA

MONTANA STATE UNIVERSITY

UNIVERSITY OF NEVADA, RENO

NEW MEXICO STATE UNIVERSITY

OREGON STATE UNIVERSITY
UNIVERSITY OF OREGON

UNIVERSITY OF SOUTHERN CALIFORNIA

STANFORD UNIVERSITY

UNIVERSITY OF HAWAII

UNIVERSITY OF TOKYO

UNIVERSITY OF UTAH

WASHINGTON STATE UNIVERSITY

UNIVERSITY OF WASHINGTON 


\section{Pacific Journal of Mathematics}

\section{Vol. 118, No. $1 \quad$ March, 1985}

Dan Amir, On Jung's constant and related constants in normed linear spaces ...1 Abdul Aziz, On the location of the zeros of certain composite polynomials . . 17 Joseph Barback, On hereditarily odd-even isols and a comparability of

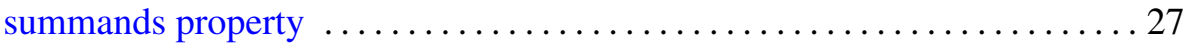

Matthew G. Brin, Klaus Johannson and Peter Scott, Totally peripheral 3-manifolds ........................................ 37

Robert F. Brown, A topological bound on the number of distinct zeros of an

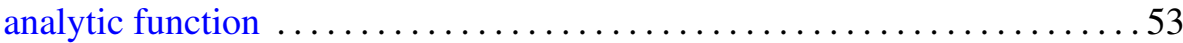

K. C. Chattopadhyay, Not every Lodato proximity is covered .......... 59

Beverly Diamond, Some properties of almost rimcompact spaces . .......63 63

Manfred Dugas and Rüdiger Göbel, On radicals and products . ......... 79

Abdelouahab El Kohen, A hyperbolic problem .................. 105

Harry Gonshor, Remarks on the Dedekind completion of a nonstandard model of the reals ................................... 117

William H. Kazez, On equivalences of branched coverings and their action

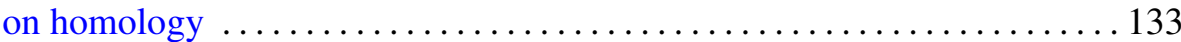

Darrell Conley Kent, On the Wallman order compactification .......... 159

Martin Andrew Magid, Lorentzian isoparametric hypersurfaces . . . . . . 165

Milan Miklavčič, Stability for semilinear parabolic equations with noninvertible linear operator

Richard Dean Neidinger and Haskell Paul Rosenthal, Norm-attainment of linear functionals on subspaces and characterizations of Tauberian operators

Johannes Vermeer, Closed subspaces of $H$-closed spaces 